\title{
Application of Lexical-Chunk-Oriented Cooperative Learning Approach to English Language Sense Cultivation
}

\author{
Shan Liu \\ School of Foreign Languages, Wuhan Textile University, Wuhan 430073, China \\ susanliu212@126.com
}

Keywords: language sense; lexical chunk; cooperative learning; cultivation modes

\begin{abstract}
Acquisition of language sense plays a fundamental role in college English education, whose effective cultivation has also become a primary concern of research. Cooperative learning is a major learning mode in college English pedagogy. Lexical chunks are community shared language constructions formulated in language use. When lexical chunks are used, speakers do not need to notice grammar structure consciously. This paper applies lexical-chunk-oriented cooperative learning approach to the cultivation of English language sense and explores its theoretical foundations and implementation modes in Listening\& Speaking and Reading\& Writing class, including retelling with lexical chunks after dictation, group discussion with lexical chunks, role-play with lexical chunks, corpus-based lexical chunk study and writing with lexical chunks, with the purpose of cultivation of language sense.
\end{abstract}

\section{Introduction}

Language sense, as defined by Tang (1999)[2], is a sense formed in the long-term access to one particular language and related culture, which is furthered illustrated as "a capability of inferring the deep meaning of speech by surface meaning in terms of discourse analysis ability." Second language sense is a logical knowledge system instead of an intuition or simply a feel, which is by no means innate (may be acquired by cultivation), cross-lingual (e.g. acquisition of English language sense does not ensure that of Japanese language sense), or permanent.

Learning of second language is a typical learning process of knowledge and skill which constructs another language system in learner's preexisting language system. Tang (2008)[4] holds that language sense of mother tongue can be developed because it is a part of language intuition, while second language sense can be learnt as an accumulating product of language knowledge. In other words, first language is endowed whereas second language ability is accomplished. The essence of second language sense is a cognitive ability, composed of concept formation, interpersonal and discourse organization ability, by quantitative learning. Concept and pragmatic functions of language sense brought about by language sense is a long continuum which accords with the features of interlanguage.

Cooperative learning is a collaborative learning with clearly defined duties and responsibilities in a group or class for a shared learning task or goal. Cooperative learning is "a group self-directed learning." (Pang, 2003)[10]. It focuses on the scaffolding and active involvement of each member to initiate interactive and negotiated learning. Cooperative language learning has become a major form of foreign language teaching with the popularity of communicative approach and task-based language learning.

\section{Background}

Researchers both in China and abroad have conducted a lot of effective studies on cooperative language learning. According to Interaction Theory by Long (1998)[7], the key to language development is interaction between learners and interaction between learners and well-designed language input material. Swain (2002) [9]believes that cooperative language learning may promote development of second language system by encouraging learners to notice the difference between their existing interlanguage system and input material by comprehensible input. Gass (2011)[5]'s experiments show that tasks which initiate a large quantities of interactions are more effective in 
promoting Spanish learning as a second language. Cooperative learning has been applied to lexical chunk learning in Jiang (2008) [6]'s study which indicates that "spoon-fed teaching should be avoided in lexical chunk teaching, while communicative approach should be adopted." In other words, cooperative tasks are largely encouraged to be designed in language teaching so that students would use chunks in interactions and communications, which results in their enhanced pragmatic ability.

College English is a language training course for the sake of second language sense cultivation which is the foundation of college English teaching. The ultimate goal for language learners is to use English and acquire some sense in language concept function, interpersonal function and discourse function. Ellis (1993) [7] points out the effective approach to foreign language learning in his paper on explicit knowledge and implicit knowledge. "Random learners tend to master the original learning material but they lack implicit knowledge and are ignorant of grammar and phrases. Rule learners could master grammar but fail to use them flexibly. Rule and instance learners may be slow starters but they can be equipped with both explicit new rules knowledge and implicit ability to judge grammar rules as they have practiced to a certain extent. "Implicit knowledge is the deep structure of language sense while explicit knowledge is the surface structure of language sense. Language sense is directly reflected in acquisition of discourse ability (listening, speaking, reading and writing). Language sense plays a critical role in discourse ability, namely, innate language competence in the way that it leads to the realization of concept function and communication function.

Cooperative learning would be effective in language sense cultivation for the following reasons. First, cooperative language learning stimulates second language learners' notice to language rules such as sentence patterns and lexical chunks. The fundamental function of language is communication function. Language sense, in essence, is the cognitive ability to language rules and concept, interpersonal and discourse analysis ability of second language. Group activities lead to the active information processing, which enables students to acquire more information and more access to actual use of language and ultimately result in the cultivation of the three abilities. Communicative demand promotes the acquisition of lexical chunks. In group activities, group members learn new lexical chunks from each other and use the newly learnt lexical chunks in authentic communication, which facilitates the process of transformation of language from input into intake. Some sentence patterns or lexical chunks can be memorized even if they occur only once in communication, which might never be possible by rote memory. Second, cooperative learning contributes to the realization of multiple conversation turns in communication, which promotes the mastery of concept function, interpersonal function and discourse function. Multiple turns could only be realized by multiple exposures to language input and involvement in language output. Language sense could never be realized by grammar learning. It is cooperative language learning that makes turn-taking in conversation possible.

\section{Implementation Strategies}

Tang (2007)[3] suggests that a large number of virtual contexts should be created for language sense cultivation so that students would drill repetitively for the purpose of mastery of three major functions of second language, namely, concept, interpersonal and discourse functions until second language sense is formed. Sentence groups should be adopted instead of single phrases and grammar analysis to enhance language sense. To be specific, in English listening and writing classes, language sense can be cultivated by combining sentence and lexical chunk input instead of single word input and cooperative language output activities. Lexical chunks are community shared language constructions formulated in language use. When lexical chunks are used, speakers do not need to notice grammar constructions consciously, giving rise to fewer grammatical mistakes and higher language accuracy and idiomaticity. "The more lexical chunks students master, the better language sense they would have, the more accurate they will express themselves in contexts and more confident they would be in using English.”(Yi, 2015)[1] 


\subsection{Language sense cultivation in Listening and Speaking}

Collaborative dialogue is an important aspect in second language learning. Collaborative dialogue means "Students support and promote each other's language learning by strategies such as questioning, solution proposing, denial, repetition, conduct activity and behavior management in their respective Zone of Proximal Development.” (Swain \& Lapkin1995)[8] Collaborative dialogue between peers is an effective strategy for language sense cultivation. Before the implementation of cooperative learning, teachers should clarify the advantages of cooperative learning and the steps to follow in implementation.

\subsubsection{Retelling with lexical chunk after dictation}

Students are divided into groups to take notes of listening material and retell. Listening materials assigned to each group could either be the same or different. If tasks between groups are the same, teachers may design some predictive questions related to listening material in preparation stage and choose some lexical chunks as the tips for questions and ask students to make predictions for the forthcoming listening material and discuss with group members with the given lexical chunks. In listening, students are required to dictate key lexical chunks. After dictation, students are required to retell and discuss the original material with given lexical chunks. In discussion, students may add new lexical chunks and finish meaning construction and retelling with the help of each other. One representative of each group would retell the passage and compete with other group representatives. Number and accuracy of lexical chunks they use in retelling would be compared. If different listening materials are given to each group, but each story fragment could construct a complete story, then information gap between groups would contribute to more meaningful communication. Then cooperative learning at a larger scale could be conducted. Groups can construct a complete story line by discussing and ranking the order of plots.

\subsubsection{Group discussion with lexical chunks}

Group discussion is a commonly used activity in spoken class. However, if insufficient language input is provided, quality of language output in group discussion is far from satisfactory with many mistakes and unidiomatic expressions. This is because all participants in group discussion are all non-native speakers whose oral English proficiency is almost the same. Language they learn from each other is limited which contributes relatively little to the interlanguage system development. If lexical chunks are given as tips for discussion questions, notices to lexical chunks are enhanced on one hand, insufficient language input is compensated on the other hand. Students' awareness of lexical chunks is enhanced in real communication.

For example, for discussion question "What would you do if one of your classmates was having a difficult time?" The following lexical chunks can be given as tips, which students are required to use as possible as they can: do my best, go through the difficulty, lose a loved one, approach them in person, show my sympathy, have financial difficulty, find a part-time job, save up.

\subsubsection{Role -play with lexical chunks}

"Foreign language is more a media, a carrier which conveys culture and values than an icon." (Tang, 2007)[3] Role play is a popular activity in spoken language learning. It offers simulated authentic situations, helps students to get rid of the dullness of language learning, adds vigor to language learning and contributes to the instilment of culture and values. Role play can be divided into full open role play which offers no tip or script, semi-open one which offers tips and closed one which offers script. Like group discussion, full open role play is likely to lead students to a wrong direction of practicing wrong expressions repetitively. Rote memory recitation of script of role play can hardly promote the improvement of language output level, either. Semi-open role play is the most effective way to facilitate accuracy and idiomaticity. In semi-open role play, teachers should clarify each group member's duties, purposely choose lexical chunks as tips for questions so as to increase chances for students to apply lexical chunks and ultimately improve accuracy and fluency of language output.

For example, for the situation that students A is staying up late for exam, while student B suggests that he should keep regular hours, the following lexical chunks are given: look upset, stay up late, get headaches, take my advice, keep regular hours, feel better. 


\subsection{Language Sense Cultivation in Reading and Writing}

In traditional reading and writing class, teachers dominate the class, which prevents the improvement of students' practical ability of using vocabulary and language output ability development as well as cultivation of language sense. Corpus-based group collaborative learning should be adopted in lexical chunk teaching. Students are divided into groups to explore on-line corpus, summarize lexical chunks in reading texts and choose typical sample sentences.

For example, for a matter of pride which appears in text, an exploration task on the usage of a matter of is given to a group of students. Student A is required to search for word collocated with $a$ matter of on its left side. Student B searches for the nouns that could be collocated with a matter of on its right. Student C searches for gerunds collocated with a matter of on its right side. Student D searches for sentences collocated with a matter of on its right side. Then each group displays their findings respectively. For example, student B finds the top five most frequently used nouns that could be collocated with a matter of are: time, urgency, concern, law and principle. He then chooses sample sentence and present his finding with other group members with PowerPoint. Teacher is supposed to evaluate each group's findings and point out their mistakes. Finally, lexical chunk output activities are designed for enhancement of cognition, such as sentence-making with lexical chunks, story-telling chain with lexical chunks, retelling with lexical chunks, changing reading text into role play script, debate etc. Students are encouraged to use newly learnt lexical chunks from texts. After activity, students are required to hand in lexical chunks list which presents chunks they have used in activity. Group which uses the most lexical chunks would be awarded.

Teachers would choose topics related to texts as writing tasks. Students are encouraged to verify the proper usage of lexical chunks with online corpus. Students are required to use many lexical chunks from texts as possible and underline them in their writing. The number and accuracy of lexical chunks are also considered as important standard for marking, which is notified to students before writing. After writing, students exchange their works with group members. They evaluate the number, accuracy of lexical chunks their partner use in writing and correct each other's mistake. Finally, teacher evaluates student's writing and makes comments on their evaluation and summarizes the wrong usages.

\section{Conclusion}

Lexical chunk-oriented cooperative learning approach is an effective strategy in language sense cultivation. Language sense could only be cultivated by recitation of a large number of lexical chunks. Teacher should also design cooperative learning tasks in listening, reading, writing for language sense cultivation. Students with individual difference learn from each other, benefit from each other, master language rules and improve language sense in cooperative learning. Future studies may offer empirical evidence for cooperative learning's effect on language sense. Predictably, cooperative learning will improve the efficiency of English learning and plays an irreplaceable role in language sense cultivation.

\section{Acknowledgement}

Supported by Chinese National Research centre for Foreign Language Education (No.zgwyjyjj2016B78) and Hubei Provincial Department of Education (No. 16Q377)

\section{References}

[1] Bing Yi. Effects of Lexical Chunk, Language Sense and Contexts on Metalinguistic Ability [J]. Journal of Huizhou College,2015(10)( in Chinese)

[2] Fuhua Tang. On Language Sense Training in CET Practice [J]. Teaching English in China, 1999 ( 1 ) ( in Chinese)

[3] Fuhua Tang, Language Sense Category and Language Strategies [J]. Journal of Xian Foreign 
Languages University,2007(6)( in Chinese)

[4] Fuhua Tang. Language sense and language intuition [J]. Foreign Language and Foreign Language Teaching,2008(4)( in Chinese)

[5] Gass, S. Task-Based Interactions in Classroom and Laboratory Settings [J]. Language Learning.2011 (5):189 - 220

[6] Yuhong Jiang. An Empirical Study on Effect of Lexical-chunk Approach on English Learners' Communicative Competence [J], Journal of International Relationship College, 2008(3):86-90( in Chinese)

[7] Long, M. H. The role of the linguistic environment in second language acquisition [J]. In W. Ritchie \& T. Bathia (eds.) Handbook of Second Language Acquisition. San Diego: academic Press,1998

[8] Swain, M. and Lapkin, S. Problems in output and the cognitive processes they generate: A step towards second language learning [J]. Applied Linguistics, 1995 (16), 371-391.

[9] Swain, M., Brooks, L. and Tocalli-Beller, A. Peer-peer dialogue as a means of second language learning [J]. Annual Review of Applied Linguistics .2002(22), 171-185

[10]Weiguo Pang. Self-directed Learning: Principle and Strategy of Learning and Teaching [M].Shanghai: Eastern China Normal University Press,2003 (in Chinese) 